\title{
Quasi-1D Compressible Flow of Hydrocarbon Fuel
}

\author{
Di Cheng ${ }^{1}$, Xuejun Fan ${ }^{2}$ and Meng Yang ${ }^{3}$ \\ Institute of Mechanics, Chinese Academy of Sciences, Beijing, P. R. China, 100190
}

\begin{abstract}
Based upon equilibrium thermodynamics, the differential equations of quasi-1D steady flow were formulated for arbitrary equation of state to study dense gas behavior of hydrocarbon fuels. A complete set of dimensionless numbers to characterize the quasi-1D dense gas dynamics is identified, classified and discussed. A new numerical method based on conservation laws is proposed to study isentropic flow and shock wave of dense gas and applied to flows of n-dodecane. Furthermore, temperature dependence of supercritical kerosene jet structure is partially interpreted by Prandtl-Meyer expansion of dense gas. It is also proved that the maximum momentum flux at the outlet of a nozzle can be achieved at Mach number $\sqrt{2}$ for isentropic flow of arbitrary fluid.
\end{abstract}

Keywords: dense gas dynamics, Supercritical hydrocarbon, underexpanded jet

\section{Nomenclature}

A. Symbol

$\begin{array}{ll}A(x) & \text { section area, }\left[\mathrm{m}^{2}\right] \\ x & \text { axial distance, }[\mathrm{m}] \\ \rho & \text { density, }\left[\mathrm{kg} / \mathrm{m}^{3}\right] \\ C_{p} & \text { specific isobaric heat capacity, }[\mathrm{J} /(\mathrm{kg} \cdot \mathrm{K})] \\ C_{v} & \text { specific isovolumetric heat capacity, }[\mathrm{J} /(\mathrm{kg} \cdot \mathrm{K})] \\ v & \text { specific volume, }\left[\mathrm{m}^{3} / \mathrm{kg}\right] \\ T & \text { temperature, }[\mathrm{K}] \\ p & \text { pressure, }[\mathrm{Pa}] \\ M & \text { Mach number } \\ c & \text { sound speed, }[\mathrm{m} / \mathrm{s}] \\ s & \text { specific entropy, }[\mathrm{J} /(\mathrm{kg} \cdot \mathrm{K})] \\ h & \text { specific enthalpy, }[\mathrm{J} / \mathrm{kg}] \\ u & \text { speed, }[\mathrm{m} / \mathrm{s}] \\ f & \text { friction coefficient } \\ \omega & \text { Prandtl-Meyer angle } \\ D & \text { hydraulic diameter, }[\mathrm{m}] \\ \delta q & \text { specific heat addition, }[\mathrm{J} / \mathrm{kg}] \\ \mu_{j}=\left(\frac{\partial T}{\partial p}\right)_{h} & \text { Joule-Thomson coefficient, }[\mathrm{K} / \mathrm{Pa}] \\ & \end{array}$

\footnotetext{
${ }^{1}$ Master Student, State Key Laboratory of High Temperature Gas Dynamics, Institute of Mechanics, Chinese Academy of Sciences, No.15 Beisihuanxi Road, Beijing, China (100190), chengdi@imech.ac.cn.

${ }^{2}$ Professor, State Key Laboratory of High Temperature Gas Dynamics, Institute of Mechanics, Chinese Academy of Sciences, No.15 Beisihuanxi Road, Beijing, China (100190), xfan@imech.ac.cn Member AIAA.

${ }^{3}$ Master Student, State Key Laboratory of High Temperature Gas Dynamics, Institute of Mechanics, Chinese Academy of Sciences, No.15 Beisihuanxi Road, Beijing, China (100190), yak1226@qq.com.
} 


$$
\begin{aligned}
& \beta=\frac{1}{v}\left(\frac{\partial v}{\partial T}\right)_{p} \quad \text { volumetric coefficient of thermal expansion, }[1 / \mathrm{K}] \\
& \kappa=-\frac{1}{v}\left(\frac{\partial v}{\partial p}\right)_{T} \text { isothermal compressibility, [1/Pa] } \\
& k_{s}=\frac{c^{2}}{p v}=\left(\frac{\partial \ln p}{\partial \ln \rho}\right)_{s} \quad \text { general isentropic index } \\
& \Gamma=\frac{v^{3}}{2 c^{2}}\left(\frac{\partial^{2} p}{\partial v^{2}}\right)_{s} \quad \text { fundamental derivative of gas dynamics } \\
& \gamma=\frac{C_{p}}{C_{v}} \quad \text { heat capacity ratio } \\
& g=\rho \mu_{j} C_{p} \quad \text { dimensionless Joule-Thomson coefficient } \\
& K=\frac{\gamma-1}{\rho C_{p} \mu_{j}+1}+1 \quad \text { equivalent heat capacity ratio } \\
& \hat{R}=C_{p}-C_{v} \quad \text { equivalent gas 'constant', }[\mathrm{J} /(\mathrm{kg} \cdot \mathrm{K})] \\
& X=\frac{\left(\partial c^{2} / \partial T\right)_{v}}{2 C_{v}}=\frac{\left(\partial c^{2} / \partial T\right)_{v}}{2(\partial e / \partial T)_{v}}=\frac{1}{2}\left(\frac{\partial c^{2}}{\partial e}\right)_{v} \quad \text { newly introduced non-dimensional number represent how sound }
\end{aligned}
$$

speed varies with internal energy.

\section{B. Subscript}

c Thermodynamically critical point properties

$0 \quad$ Isentropic stagnation properties

$r \quad$ Reduced parameters, normalized by critical properties

\section{Superscript \\ * Throat parameters}

\section{Introduction}

DEAL gas dynamics is limited by its ideal gas assumption, which confines its accuracy and validity to high temperature and low pressure gas condition. Its applicability to arbitrary fluid's compressible flow in arbitrary temperature and pressure range is in doubt and must be verified.

In the same time, for its special properties such as high density, low viscosity and low sound speed, supercritical fluid is widely used in industries, such as active cooling in scramjet, ${ }^{1,2}$ high pressure steam turbine, organic Rankine cycle etc. due to its moderate critical pressure and temperature, and low sound speed as Figure 1 shows. ${ }^{3}$ It is easy to accelerate supercritical hydrocarbon fuel to supersonic in near-critical region. Another benefits of supercritical hydrocarbon fuel is that they are dry fluid if we regard it as working fluid of an incomplete supercritical Rankine cycle, as Figure 7(a) shows, which means it will not condense during isentropic expansion and avoid some problems associated with two-phase flow. ${ }^{4}$ However, the flow characteristics of supercritical fluid's compressible flow are complicated. It cannot be treated as ideal gas because compressible factor's deviation from one, or be regarded as incompressible fluid due to its low sound speed. So we need to deal with it using dense gas dynamics (someone call it dense gas dynamics or real gas dynamics) without presuming the form of its equation of state. 


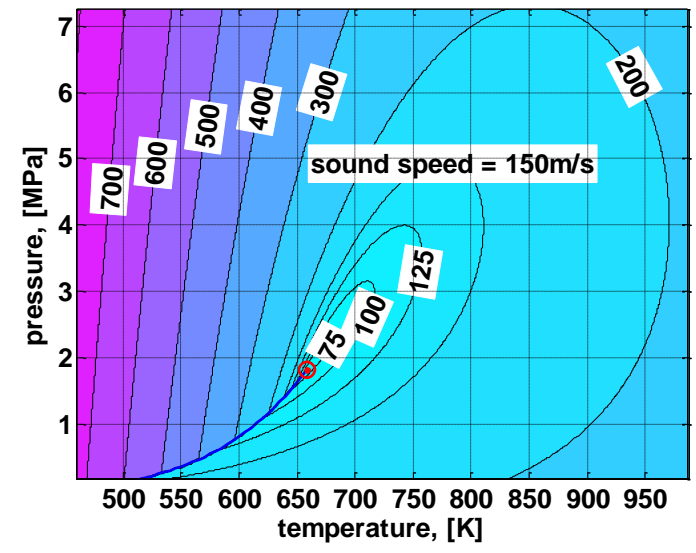

Figure 1 sound speed contour of $n$-dodecane in $p-T$ plane.

According to the literatures, study of dense gas dynamics could be traced back to P. A. Thompson's work, ${ }^{5}$ which introduced fundamental derivative of gas dynamics and discussed the its importance in compressible flow. D. A. Kouremenos summarized three real gas isentropic exponents and introduced the general isentropic exponents $k_{s}$ to describe the behavior of real gas flow. ${ }^{6}$ M. Cramer et al. formulated exact solution of Van der Waals gas' isentropic flow and numerically solved isentropic flow using Martin-Hou equations of state. ${ }^{7}$ Besides, G. H. Schnerr et al. deduced formulae for quasi-1D flow with variable section area and heat addition. ${ }^{8}$ They even get a exact solution for transonic flow of dense gases. ${ }^{9}$ D. Stojkovic et al. studied the effect of friction in steady flow of dense gases in pipes. ${ }^{10}$ Those studies reveal the importance of $\Gamma$ in isentropic flow of real fluid and predicted some particular phenomena in dense gas flow. ${ }^{11}$ However, the formulae deduced in those studies are not so compact and not fully simplified, or oversimplified to certain equation of state, which limits their application.

Recently, several groups in Europe, such as Prof. P. M. Colonna in TU Delft, Prof. A. Guardone at Politecnico di Milano, focused on studying Bethe-Zel'dovich-Thompson fluid, ${ }^{12}{ }^{13}$ which has negative fundamental derivative of gas dynamics and will not produce shock in compression. They developed numerical methods to solve real fluid's compressible flow, ${ }^{14}$ and built the flexible asymmetric shock tube (FAST) for dense gas experiments. ${ }^{15}$ They are interested in organic Rankine cycle utilizing a family of siloxane as working fluid, ${ }^{16}$ which has a large area of negative $\Gamma$ near the critical point. However, hydrocarbon fuels do not have large $\Gamma<0$ region but a large $0<\Gamma<1$ region near the critical point. The conclusion they drew might be different in supercritical hydrocarbon fuels.

In Section II, Based upon equilibrium thermodynamics and Bridgman's table,$^{17}$ we deduced the differential equations of single phase, quasi-1D, steady flow for arbitrary fluid, and we found a complete set of dimensionless number $g, k_{s}, \gamma, \Gamma, X$ to characterize the dense gas dynamics formulae. Then we proved that $\left(\rho u^{2}\right)=\left(\rho u^{2}\right)_{\max }$ when $M=\sqrt{2}$ for arbitrary fluid in isentropic flow. Next, the relation between the variation of total temperature after shockwave and the Joule-Thomson coefficient is proved. In Section III, numerical methods were developed, combined with SUPERTRAPP thermodynamics software, ${ }^{18}$ to simulate the supercritical hydrocarbon fuel's isentropic flow through Laval nozzle with shockwave. N-dodecane is selected as a surrogate for the sake of conciseness. In Section IV, Prandtl-Meyer expansion wave theory combined with above dense gas dynamics is used to interpret the underexpanded supercritical kerosene jet. At last, conclusions are summarized in Section V.

\section{Theoretical analysis}

\section{A. Fundamental equations}

Conservation of mass:

$$
\rho u A=\text { const } \Rightarrow-\frac{d v}{v}+\frac{d u}{u}+\frac{d A}{A}=0
$$

First law of thermodynamics:

$$
d\left(h+u^{2} / 2\right)=d h+u d u=v d p+T d s+u d u=\delta q
$$

Second law of thermodynamics:

$$
T d s=\delta q+\frac{2 f u^{2}}{D} d x
$$


Equation of state, generalized differential form:

$$
\begin{gathered}
p=p(v, s) \Rightarrow d p=-\frac{c^{2}}{v^{2}} d v+\left(\frac{\partial p}{\partial s}\right)_{v} d s=-\frac{c^{2}}{v^{2}} d v-\left(\frac{\partial T}{\partial v}\right)_{s} d s \\
T=T(v, s) \Rightarrow d T=\left(\frac{\partial T}{\partial v}\right)_{s} d v+\left(\frac{\partial T}{\partial s}\right)_{v} d s
\end{gathered}
$$

We did not use momentum equation because it can be deduced using (2) and (3) in this case.

\section{B. First order derivative related formulae}

Definition of sound speed:

$$
c^{2}=\left(\frac{\partial p}{\partial \rho}\right)_{s}=v^{2} \cdot\left(\frac{\partial p}{\partial v}\right)_{s}=\gamma \frac{v}{\kappa}=\gamma\left(C_{p}-C_{v}\right) T \cdot\left(\frac{v}{\mu_{j} C_{p}+v}\right)^{2}=\gamma \hat{R} T \cdot\left(\frac{1}{g+1}\right)^{2}
$$

Using Bridgman's Table to convert first order derivatives to $C_{p}, C_{v}, \mu_{j}$ :

$$
\begin{gathered}
\left(\frac{\partial T}{\partial v}\right)_{s}=-\frac{C_{p}-C_{v}}{C_{v}} \frac{T}{\mu_{j} C_{p}+v}=-(K-1) T / v \\
\left(\frac{\partial T}{\partial s}\right)_{v}=\frac{T}{C_{v}}
\end{gathered}
$$

Utilizing the symbols defined in the nomenclature, the equations above are used to deduce the differential relations for $d p, d T, d v, d u$ as below:

$$
\begin{gathered}
\frac{d p}{p}=k_{s} \cdot \frac{M^{2}}{1-M^{2}} \frac{d A}{A}-k_{s} \cdot \frac{(K-1) M^{2}}{1-M^{2}} \cdot \frac{\delta q}{c^{2}}-k_{s} \cdot \frac{M^{2}}{2} \cdot \frac{(K-1) M^{2}+1}{1-M^{2}} \cdot \frac{4 f d x}{D} \\
\frac{d T}{T}=\frac{(K-1) M^{2}}{1-M^{2}} \frac{d A}{A}+\frac{(K-1)^{2}}{\gamma-1} \frac{1-\gamma M^{2}}{1-M^{2}} \frac{\delta q}{c^{2}}+\frac{M^{2}}{2} \frac{(K-1)\left(K-\gamma-\gamma(K-1) M^{2}\right)}{(\gamma-1)\left(1-M^{2}\right)} \frac{4 f d x}{D} \\
-\frac{d \rho}{\rho}=\frac{d v}{v}=\frac{d u}{u}+\frac{d A}{A}=-\frac{M^{2}}{1-M^{2}} \frac{d A}{A}+\frac{K-1}{1-M^{2}} \frac{\delta q}{c^{2}}+\frac{K M^{2}}{2\left(1-M^{2}\right)} \frac{4 f d x}{D} \\
\frac{d u}{u}=-\frac{1}{1-M^{2}} \frac{d A}{A}+\frac{(K-1)}{1-M^{2}} \frac{\delta q}{c^{2}}+\frac{K M^{2}}{2\left(1-M^{2}\right)} \frac{4 f d x}{D}
\end{gathered}
$$

In which the equivalent heat capacity ratio $K$ and general isentropic exponent $k_{s}$ are defined as:

$$
\begin{aligned}
& K=\frac{\gamma-1}{\rho C_{p} \mu_{j}+1}+1 \\
& k_{s}=\frac{c^{2}}{p v}=\left(\frac{\partial \ln p}{\partial \ln \rho}\right)_{s}
\end{aligned}
$$

The detailed deduction is attached in Appendix A.

From those equations deduced, at least three conclusions could be drawn:

1. Equivalent heat capacity ratio $K$ could replace $\gamma$ in many cases. And $K \rightarrow \gamma$ when $\mu_{j} \rightarrow 0$. So we can conclude that when $\mu_{j} \rightarrow 0$, the quasi-1D differential relationships of ideal gas dynamics become more valid for dense gas flow, and the deviation depends on $\mu_{j}$.

2. Velocity and density variations with respect to area change only depend on Mach number and are independent of fluid property. So the the variation of momentum flux or dynamic pressure is only depend on Mach number and area change.

3. According to Bridgman's Table, $K-1=\frac{\gamma-1}{\rho \mu_{j} C_{p}+1}=\frac{\beta}{\rho \kappa C_{v}}$, and because $\kappa>0, \gamma>1$ is valid for any thermodynamically stable system, so the $\frac{d T}{d A}, \frac{d p}{\delta q}, \frac{d u}{\delta q}$ might be different from the prediction of ideal gas dynamics when $\beta<0$. However, according to the available thermodynamic data, $\beta<0$ only occurs in low temperature liquid region in which it shows little compressibility. 


\section{Second order derivative related formulae}

Introducing the definition of fundamental derivative of gas dynamics,

$$
\Gamma=\frac{v^{3}}{2 c^{2}}\left(\frac{\partial^{2} p}{\partial v^{2}}\right)_{s}=1+\frac{\rho}{c}\left(\frac{\partial c}{\partial \rho}\right)_{s}
$$

And a new non-dimensional number $\mathrm{X}$ defined as:

$$
X=\frac{\left(\partial c^{2} / \partial T\right)_{v}}{2 C_{v}}=\frac{\left(\partial c^{2} / \partial T\right)_{v}}{2(\partial e / \partial T)_{v}}=\frac{1}{2}\left(\frac{\partial c^{2}}{\partial e}\right)_{v}
$$

We can write the complete differential form of sound speed as below:

$$
\frac{d c}{c}=(1-\Gamma) \frac{d v}{v}+X \frac{T d s}{c^{2}}
$$

Substitute Eq.(11) and Eq.(3), we got

$$
\frac{d c}{c}=\frac{M^{2}(\Gamma-1)}{1-M^{2}} \frac{d A}{A}+\left(\frac{(K-1)(1-\Gamma)}{1-M^{2}}+X\right) \frac{\delta q}{c^{2}}+\frac{M^{2}}{2}\left(\frac{K(1-\Gamma)}{\left(1-M^{2}\right)}+X\right) \frac{4 f d x}{D}
$$

Using the definition of Mach number

$$
M=\frac{u}{c} \Rightarrow \frac{d M}{M}=\frac{d u}{u}-\frac{d c}{c}
$$

Mach number's variation could be derived as:

$$
\frac{d M}{M}=-\frac{1+M^{2}(\Gamma-1)}{1-M^{2}} \frac{d A}{A}+\left(\frac{(K-1) \Gamma}{1-M^{2}}-X\right) \frac{\delta q}{c^{2}}+\frac{M^{2}}{2}\left(\frac{K \Gamma}{\left(1-M^{2}\right)}-X\right) \frac{4 f d x}{D}
$$

From those equations deduced, at least three conclusions could be drawn:

1. When the equation of state approaches calorically perfect gas' model, we can get

$$
K \rightarrow \gamma, \frac{c^{2}}{p v} \rightarrow \gamma, \frac{\delta q}{c^{2}} \rightarrow \frac{1+\frac{\gamma-1}{2} M^{2}}{\gamma-1} \cdot \frac{d T_{0}}{T_{0}}, X=\frac{\left(\partial c^{2} / \partial T\right)_{v}}{2 C_{v}} \rightarrow \frac{\gamma(\gamma-1)}{2}, \Gamma \rightarrow \frac{(\gamma+1)}{2}
$$

Then the Eqs.(20), (18), (9), (10), (11) and (12) can be reduced to the classical form of differential relations of perfect gas dynamics. ${ }^{19}$

2. Considering isentropic flow without heat addition and friction, if $\Gamma<1$, sound speed varies different with ideal gas dynamics theory's prediction. Because for ideal gas,

$$
\Gamma=\frac{\gamma+1}{2}+\frac{\gamma-1}{2 \gamma} T \frac{d \gamma}{d T}
$$

To normal gases showing ideal gas behavior, $\gamma$ do not vary fast with $T$, so $\Gamma \approx \frac{\gamma+1}{2}>1$.

For most fluid, especially major components in hydrocarbon fuels, $\Gamma<1$ region is near critical point and attached to saturated vapor curve, as Figure 2 shows.

3. Step further, if $\Gamma<1-1 / M^{2}$, Mach number varies differently with ideal gas theory in isentropic flow without heat and addition and friction. For instance, in supersonic flow via diverging nozzle, Mach number might decrease, as many precedent literature Numerical Case $\mathrm{C}$ shows. ${ }^{7}$ 


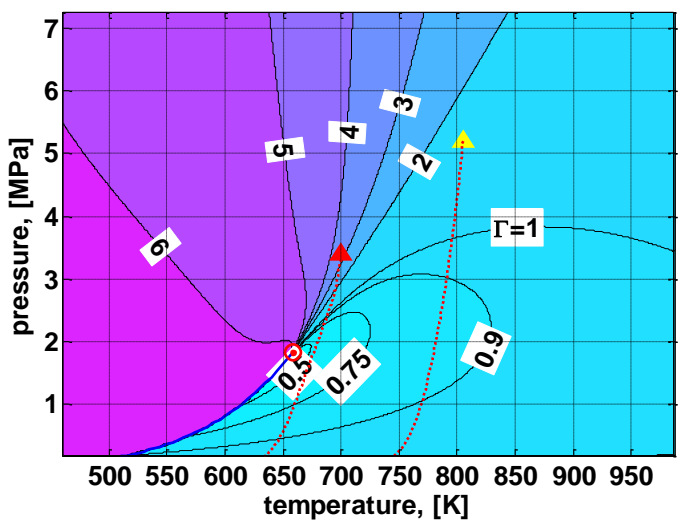

Figure 2. The contour of the fundamental derivative of gas dynamics of n-dodecane. it is calculated utilizing SUPERTRAPP.

\section{Hierarchy of non-dimensional parameters and thermodynamic derivatives}

According to the Bridgman's article, ${ }^{17}$ only three of all first order thermodynamic derivatives of $e, h, f, g, T, s, p, v$ with respect to each other is independent, and the completeness of second order derivatives need only two extra independent second order derivatives. According to the formulae deduced, $1^{\text {st }}$ and $2^{\text {nd }}$ order thermodynamic derivatives are sufficient to describe the flow of dense gas as shown in Table 3, except that in transonic region where $3^{\text {rd }}$ or $4^{\text {th }}$ order thermodynamic derivatives are needed. ${ }^{9}$ The non-dimensional parameters used in the equations above are summarized in Table 1 and the thermodynamic derivatives are grouped into $0^{\text {th }}, 1^{\text {st }}$ and $2^{\text {nd }}$ orders.

Table 1. Hierarchy of non-dimensional parameters of dense gas flow.

\begin{tabular}{|c|c|c|c|}
\hline \multirow{4}{*}{\begin{tabular}{l}
\multicolumn{1}{c}{ Dynamics } \\
Mach number: \\
$M=u / c$
\end{tabular}} & & Thermodynamics & Chemical Equilibrium \\
\hline & $0^{\text {th }}$ order & Compressibility factor: $Z=p v / R T$ & \multirow{3}{*}{$\begin{array}{l}\text { Fugacity coefficient: } \\
\phi_{i}=f_{i} / p_{i}\end{array}$} \\
\hline & $1^{\text {st }}$ order & $\begin{array}{l}\text { Heat capacity ratio: } \gamma=C_{p} / C_{v} \\
\text { General isentropic exponent: } \\
k_{s}=c^{2} / p v=\left(\frac{\partial \ln p}{\partial \ln \rho}\right)_{s} \\
\text { Non-dimensional Joule-Thompson Coeff.: } \\
g=\rho C_{p} \mu_{j}=\rho C_{p}\left(\frac{\partial T}{\partial p}\right)_{h}\end{array}$ & \\
\hline & $2^{\text {nd }}$ order & $\begin{array}{l}\text { Fundamental derivative of gas dynamics: } \\
\Gamma=\frac{v^{3}}{2 c^{2}}\left(\frac{\partial^{2} p}{\partial v^{2}}\right)_{s}=1+\rho c\left(\frac{\partial c}{\partial p}\right)_{s}=\frac{1}{c}\left(\frac{\partial(\rho c)}{\partial \rho}\right)_{s} \\
\text { New non-dimentional number: } \\
X=\frac{\left(\partial c^{2} / \partial T\right)_{v}}{2 C_{v}}=\frac{\left(\partial c^{2} / \partial T\right)_{v}}{2(\partial e / \partial T)_{v}}=\frac{1}{2}\left(\frac{\partial c^{2}}{\partial e}\right)_{v}\end{array}$ & \\
\hline
\end{tabular}

The physical interpretation of every non-dimensional parameter is listed below:

- Mach number $M$ : the compressibility of the flow.

- Compressibility factor $Z$ : the compressibility of the fluid itself. It is convenient to use it to relate $p, v$ and $T$. But it is not so convenient to calculate fluid flow.

- Heat capacity ratio $\gamma$ : the ideal gas part of its thermodynamic properties. It indicates the significance of the energy needed to expand. It is always greater than one.

- General isentropic exponent $k_{s}$ : isentropic compressibility property. It relates the sound velocity with $p v$. It appears only at $d p / p$ term, which means it indicates the discrepancy of pressure changes from that of ideal gas flow. 
- Non-dimensional J-T coefficient $g$ : some dense gas part of its thermodynamic properties.

- Fundamental derivative of gas dynamics $\Gamma$ : how sound velocity changes during isentropic expansion.

- New non-dimensional number $X$ : how sound speed changes with internal energy.

- Fugacity coefficient $\phi_{i}$ : chemically effective part of partial pressure. Chemical equilibrium will be very different from that at low pressure.

All the non-dimensional numbers' value of dodecane are plotted on the pressure-temperature plane in Figure 11 at the Appendix.

\section{E. Maximum jet momentum flux}

Fuel jet into supersonic cross flow is an important phenomenon in scramjet combustor. The jet penetration is primarily determined by the momentum flux $\rho u^{2}$ of fuel jet. ${ }^{20,21}$ Assuming the cross jet is a quasi-1D process, we can write the variation of $\rho u^{2}$ as:

$$
\frac{d\left(\rho u^{2}\right)}{\rho u^{2}}=2 \frac{d u}{u}-\frac{d v}{v}=\frac{M^{2}-2}{1-M^{2}} \frac{d A}{A}+\frac{K-1}{1-M^{2}} \frac{\delta q}{c^{2}}+\frac{K M^{2}}{2\left(1-M^{2}\right)} \frac{4 f d x}{D}
$$

Usually, the flow via transportation tubes is subsonic and section area does not change. Most heat transfer and friction occurs in the transportation tubes. Assuming $g>-1 \Leftrightarrow K-1>0$, which is valid for supercritical and vaporized hydrocarbon fuels, heat addition and friction will increase the momentum flux density.

Considering flow via Laval nozzle connecting transportation tubes to combustion chamber, if we can neglecting friction and heat transfer, we can conclude that

$$
\begin{cases}d A<0, M<1 & \Rightarrow d\left(\rho u^{2}\right)>0 \\ d A>0, \sqrt{2}>M>1 & \Rightarrow d\left(\rho u^{2}\right)>0 \\ d A>0, M>\sqrt{2} & \Rightarrow d\left(\rho u^{2}\right)<0\end{cases}
$$

It means that $M=\sqrt{2}$ is the local extremum of $\rho u^{2}$. If the Mach number increases in diverging section of Laval nozzle monotonously, $M=\sqrt{2}$ is the global maximum. However, it might be either local maximum or minimum in some case, depending on the change of Mach number in diverging section of Laval nozzle, as Numerical Case C shows.

Comparing with $M=1$ nozzle, $M=\sqrt{2}$ nozzle could get about $15 \%$ extra outlet momentum flux density using supercritical n-dodecane, as Numerical Case A shows, which will increase approximately $6 \%$ jet penetration depth according to formula of Billig et al. 's work as shown in Eq.(25). ${ }^{22}$ And the improvement is nearly costless.

$$
\frac{P}{D}=\left(\frac{q_{\text {jet }}}{q_{\text {air }}}\right)^{0.435} \times\left(\frac{x}{D}\right)^{0.435}
$$

In which $P$ denotes penetration depth. $D$ denotes injector orifice diameter. $q_{a i r}, q_{j e t}$ denote the dynamic pressure of air and fuel jet respectively. $x$ is the streamwise distance from the injector.

For calorically perfect ideal gas, area ratio of Mach $\sqrt{2}$ outlet and throat is as below.

$$
\frac{A_{M=\sqrt{2}}}{A^{*}}=\frac{\sqrt{2}}{2}\left(\frac{2 \gamma}{\gamma+1}\right)^{\frac{\gamma+1}{2(\gamma-1)}}
$$




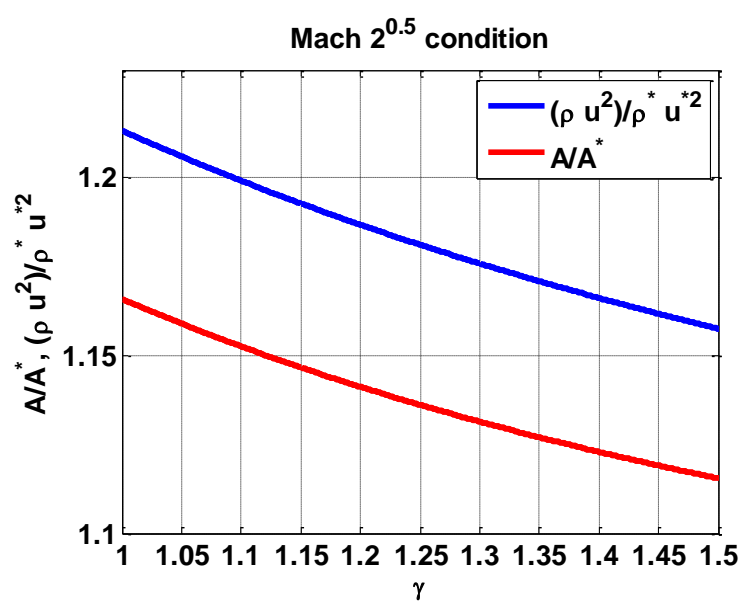

Figure 3 Area ratio of outlet and throat of Mach $\sqrt{2}$ nozzle for perfect gas.

\section{F. Stagnation properties after shock wave}

In this paper, the stagnation properties are defined as below:

$$
\begin{aligned}
& T_{0}=T_{0}\left(h_{0}, s_{0}\right)=T\left(h+u^{2} / 2, s\right) \\
& p_{0}=p_{0}\left(h_{0}, s_{0}\right)=p\left(h+u^{2} / 2, s\right)
\end{aligned}
$$

After shock wave, entropy will increase, but stagnation enthalpy will not change. So we can study how $p_{0}, T_{0}$ changes across shock wave using thermodynamic derivatives as below:

$$
\begin{gathered}
\left(\frac{\partial p}{\partial s}\right)_{h}=-\frac{T}{v}<0 \\
\left(\frac{\partial T}{\partial s}\right)_{h}=\left(\frac{\partial T}{\partial p}\right)_{h}\left(\frac{\partial p}{\partial s}\right)_{h}=-\frac{T \mu_{j}}{v}
\end{gathered}
$$

It shows that stagnation pressure always decrease after shock wave, as showed in Thompson's book. ${ }^{11}$ And the stagnation temperature's change depends on the sign of Joule-Thomson coefficient $\mu_{j}$, if $\mu_{j}$ do not change sign on the iso-enthalpy line connecting before-shock and after-shock stagnation status, as the Kouremenos showed. ${ }^{6}$ According to the generalized Joule-Thompson inversion curve for several corresponding states fluids, in the working range of supercritical kerosene (above 1.1 $T_{c}$ and about 1.5 2.0 $P_{c}$ ), the $\mu_{j}$ is always positive. ${ }^{23}$

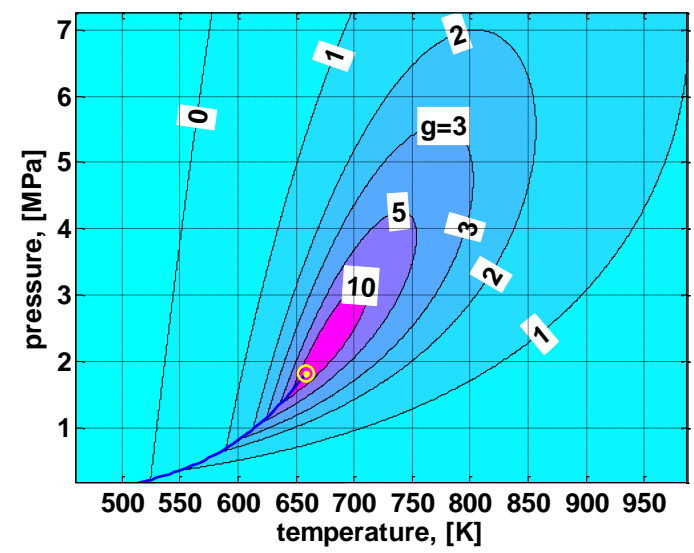

Figure $4 \mathrm{~g}=\rho \mu_{j} C_{p}$ contour of n-dodecane.

Entropy and $C_{p}$ share the same unit, so we can normalize Eq.(30) by $T$ and $C_{p}$ :

$$
\frac{C_{p}}{T}\left(\frac{\partial T}{\partial s}\right)_{h}=-\rho \mu_{j} C_{p}=-g
$$

It shows the significance of dimensionless Joule-Thomson coefficient in dense gas flow. 


\section{Numerical simulation}

We've tried to use an ODE solver to solve the differential equations deduced above, but the output cannot keep conservation of entropy and total enthalpy because of accumulation of numerical errors. So we designed a kind of new algorithm based on the conservation laws to guarantee the validity of the solution. N-dodecane is used as a surrogate of kerosene in this chapter because of the inaccuracy of current kerosene surrogate models and the accuracy of equation of state of dodecane.

\section{A. Isentropic flow}

Define unknown parameter vector $\mathbf{x}$ and conservation parameter vector $\mathbf{y}$ :

$$
\begin{gathered}
\mathbf{x}=[u, p, T]^{\prime} \\
\mathbf{y}=\left[u A / v, s, h+u^{2} / 2\right]^{\prime}
\end{gathered}
$$

Then we have the nonlinear equations as below:

$$
\mathbf{y}\left(\mathbf{x}_{1} ; A_{1}\right)=\mathbf{y}\left(\mathbf{x}_{2} ; A_{2}\right)=\text { const }
$$

We used Newton-Ralphson iteration method to solve the nonlinear equation. The Jacobian matrix is given as below:

$$
\mathbf{J}=\left[\begin{array}{ccc}
\frac{A}{v} & -\frac{u A}{v^{2}}\left(\frac{\partial v}{\partial p}\right)_{T} & -\frac{u A}{v^{2}}\left(\frac{\partial v}{\partial T}\right)_{p} \\
0 & \left(\frac{\partial s}{\partial p}\right)_{T} & \left(\frac{\partial s}{\partial T}\right)_{p} \\
u & \left(\frac{\partial h}{\partial p}\right)_{T} & \left(\frac{\partial h}{\partial T}\right)_{p}
\end{array}\right]
$$

Again, we convert all first order thermodynamic derivatives to $\mu_{j}, C_{p}, C_{v}$ using Bridgman's Table. The $\mu_{j}, C_{p}, C_{v}$ can be calculated using SUPERTRAPP given $p, T$.

This method can guarantee that the flow is isentropic and adiabatic if the thermodynamic software is accurate sufficiently.

However, this method cannot be used to solve transonic part of the flow due to zero of the Jacobian at $M=1$ :

$$
|\mathbf{J}|=-\frac{A C_{p}\left(1-M^{2}\right)}{T}
$$

So the convergence at $M=1$ is not guaranteed. And the data in transonic area is obtained via interpolation. The result is plotted in Figure 5. And the stagnation point and the isentropic line are plotted on the Figure 4. 

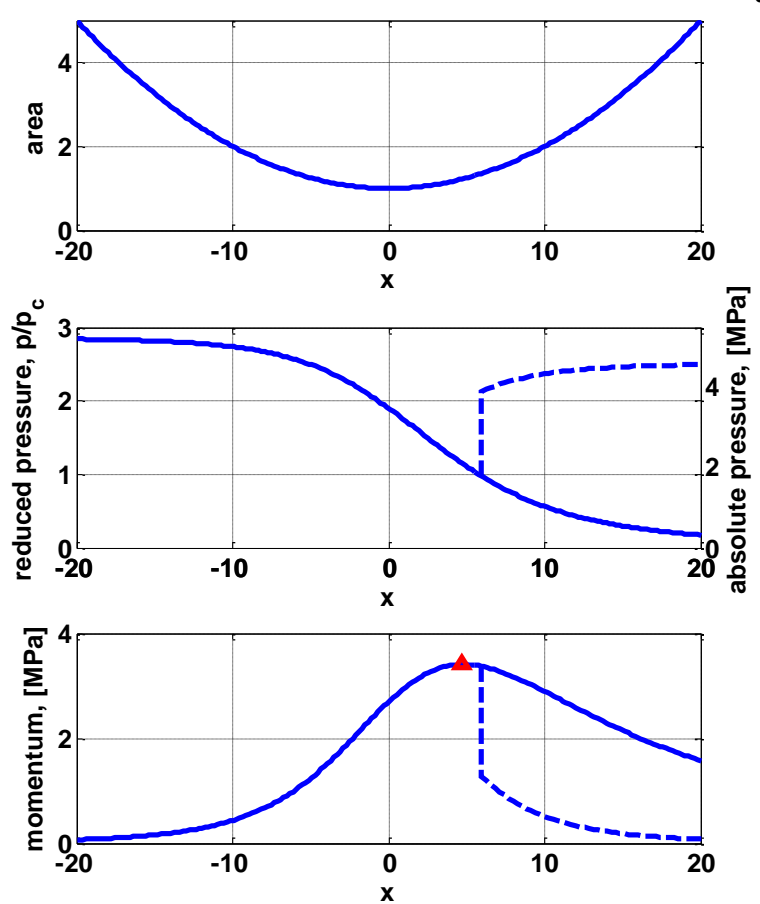
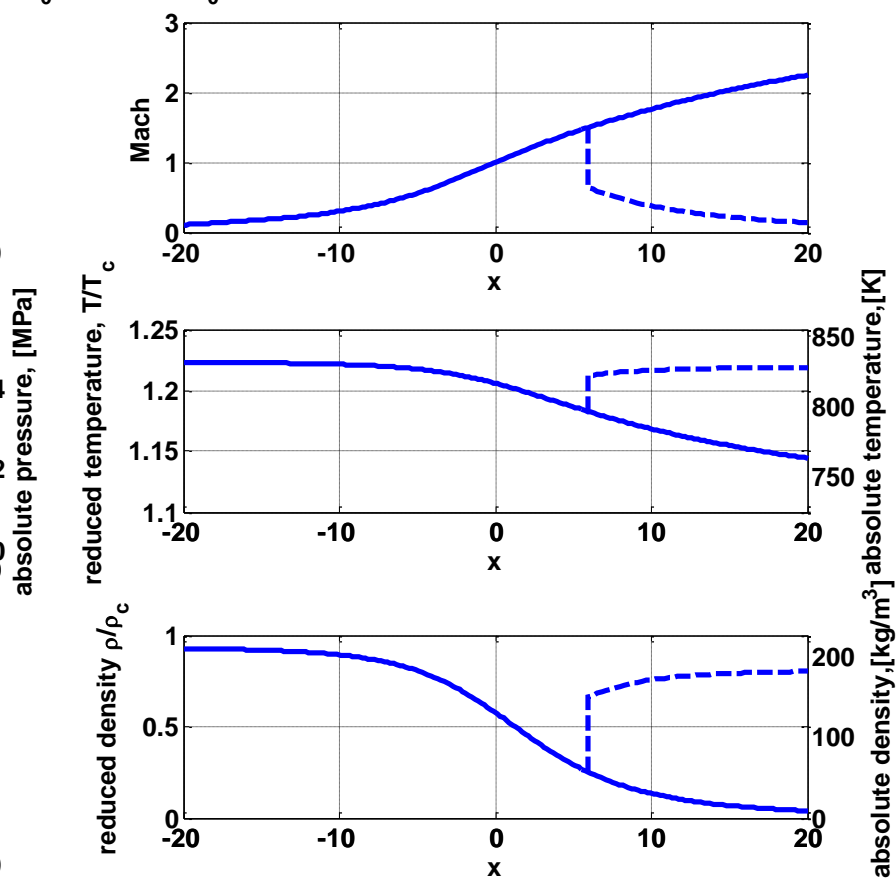

Figure 5 isentropic flow of n-dodecane in Laval nozzle. Solid line is continuous flow. dotted line is shock wave and after shock flow. Red triangle is the maximum momentum flux density.

\section{B. Shock wave}

As the isentropic flow, we define flow parameter vector $\mathbf{x}$ and conservation parameter $\mathbf{y}_{\text {shock }}$ :

$$
\begin{gathered}
\mathbf{x}=[u, p, T]^{\prime} \\
\mathbf{y}_{\text {shock }}=\left[u A / v, p+u^{2} / v, h+u^{2} / 2\right]^{\prime}
\end{gathered}
$$

And the Jacobian is like this:

$$
\mathbf{J}_{\text {shock }}=\left[\begin{array}{ccc}
\frac{A}{v} & -\frac{u A}{v^{2}}\left(\frac{\partial v}{\partial p}\right)_{T} & -\frac{u A}{v^{2}}\left(\frac{\partial v}{\partial T}\right)_{p} \\
u & 1-\frac{u^{2}}{v^{2}}\left(\frac{\partial v}{\partial p}\right)_{T} & -\frac{u^{2}}{v^{2}}\left(\frac{\partial v}{\partial T}\right)_{p} \\
u & \left(\frac{\partial h}{\partial p}\right)_{T} & \left(\frac{\partial h}{\partial T}\right)_{p}
\end{array}\right]
$$

The determinant of $\mathbf{J}_{\text {shock }}$ shows that the zero only occurs at $M=1$. And this Newton-Ralphson method seems always converging to a solution. To weak shock wave and $\Gamma>0$ condition, it can be shown that the shockwave solution exists and is unique. ${ }^{11}$ Furthermore, because Newton-Ralphson method is a second order algorithm, it should converge faster than algorithms based upon fixed point iteration metioned in literatures. ${ }^{24,25}$

We used this algorithm to calculate a $M_{\text {beforeshock }}=1.5$ shock wave. The result shows in Figure 5 . The decrease of stagnation temperature was observed as Table 2. But the change of stagnation temperature is insignificant in this case. 
Table 2 stagnation properties of Numerical Case B before and after shock wave

\begin{tabular}{ccc}
\hline & Before shock & After shock \\
\hline$p_{0}$ & $5.2 \mathrm{MPa}$ & $4.57 \mathrm{MPa}$ \\
$T_{0}$ & $805 \mathrm{~K}$ & $802.5 \mathrm{~K}$ \\
\hline
\end{tabular}

\section{Non-monotonous Mach number variation during expansion}

As mentioned previously, Mach number will reduce in supersonic expansion if $\Gamma<1-1 / M^{2}$ is fulfilled locally. According to Figure 2, we noticed that $\Gamma=0.75$ line is located around $700 \mathrm{~K}, 2.5 \mathrm{MPa}$. Considering isentropic line is nearly parallel to isothermal line for supercritical $\mathrm{n}$-dodecane, we calculated flow via Laval nozzle with $p_{0}=3.4 \mathrm{MPa}$, $T_{0}=700 \mathrm{~K}$. The result is as Figure 6 .

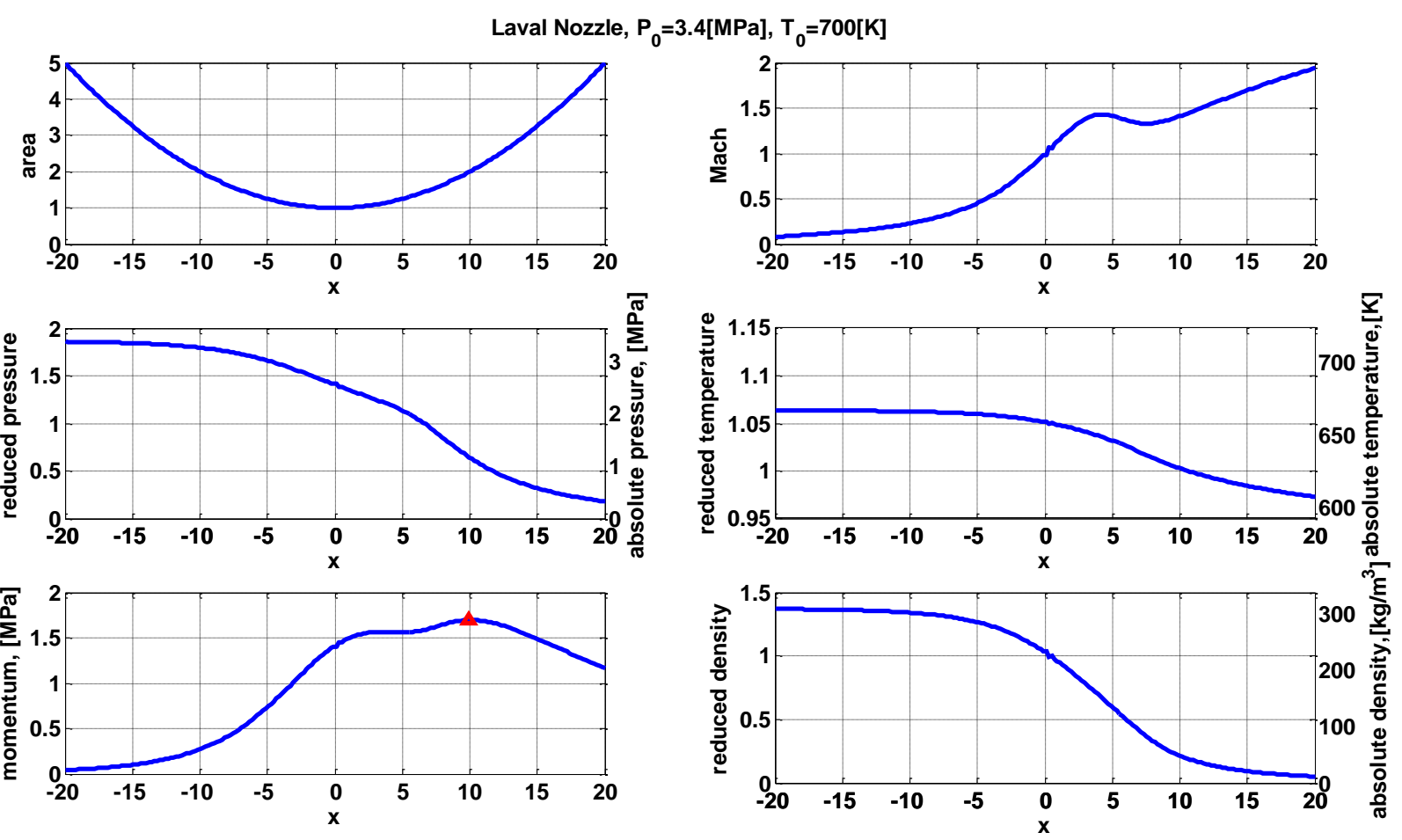

Figure 6 Flow via Laval nozzle with $p_{0}=3.4 \mathrm{MPa}, T_{0}=700 \mathrm{~K}$. Due to its non-monotonous behavior, Mach number crosses the $\sqrt{2}$ three times, corresponding to local maximum, local minimum and global maximum separately.

Two important phenomena are observable here:

1. Non-monotonous Mach number variation during expansion in the diverging section.

2. Three extremum of momentum flux density along the axis, all located at $M=\sqrt{2}$.

Those results might be utilized in two ways:

1. If you want to avoid dense gas behavior, a higher $T_{0}$ must be chosen.

2. When designing Mach $\sqrt{2}$ nozzle, all $M=\sqrt{2}$ points should be analyzed to select the best.

\section{Underexpanded jet structure of supersonic kerosene}

The application of the formula above are limited by quasi-1D assumption, which results in the difficulty to verify it experimentally, because no measurable quantity changes against the prediction of ideal gas dynamics qualitatively 
in quasi-1D isentropic flow. However, the theory can be extended to 2D or axisymmetric condition easily with Prandtl-Meyer expansion wave theory, which is verifiable using the shape of supersonic free jet.

So we tried to interpret the sonic free jet experimental results obtained by Meng Yang, as showed in Figure 9. ${ }^{26}$ Because the kerosene is dry fluid, as shown in Figure 7, condensation during isentropic expansion is not admissible.

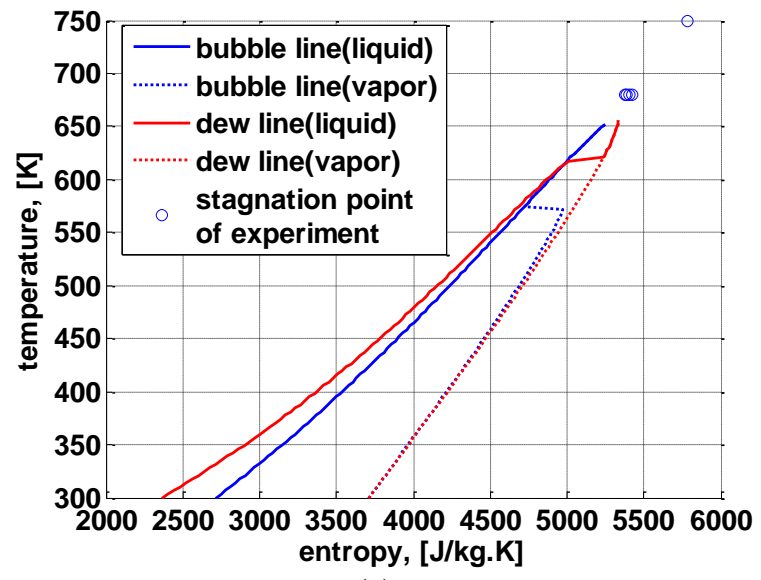

(a)

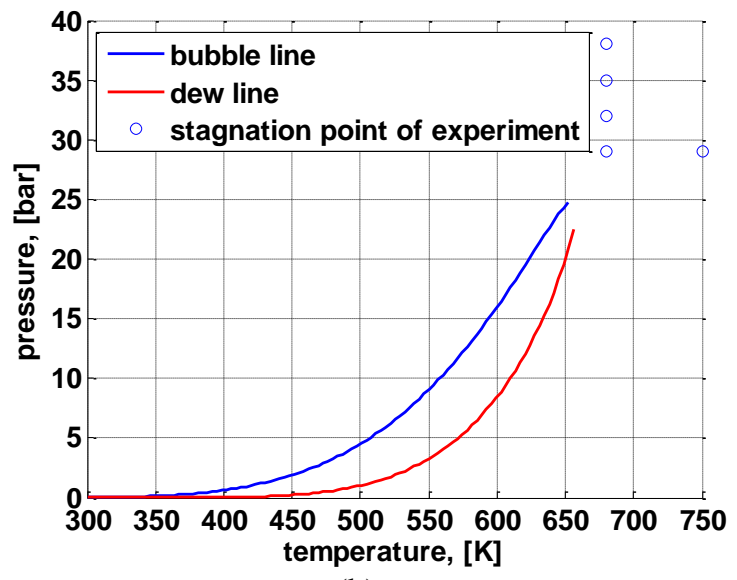

(b)

Figure 7 (a) T-s diagram of kerosene produced using SUPERTRAPP program. (b) Saturation curve of kerosene on the p-T diagram. The critical region cannot be resolved by SUPERTRAPP. The 10 species surrogate of China RP-3 kerosene is obtained from literature. ${ }^{27}$ The critical point of RP-3 kerosene is $645.04 \mathrm{~K}$ and 23.4 bar. ${ }^{28}$

Further assuming the jet process is isentropic, so the Prandtl-Meyer's theory can be applied to calculate the jet angle at the edge of the orifice using the formulae below. ${ }^{11}$

$$
d \omega=\sqrt{M^{2}-1} \frac{d u}{u}
$$

The boundary condition is:

$$
\left.\omega\right|_{M=1}=0
$$

Again the dodecane is used as a surrogate, integeration along isentropic line results in $\omega(u)$ function as Figure 8 shows.

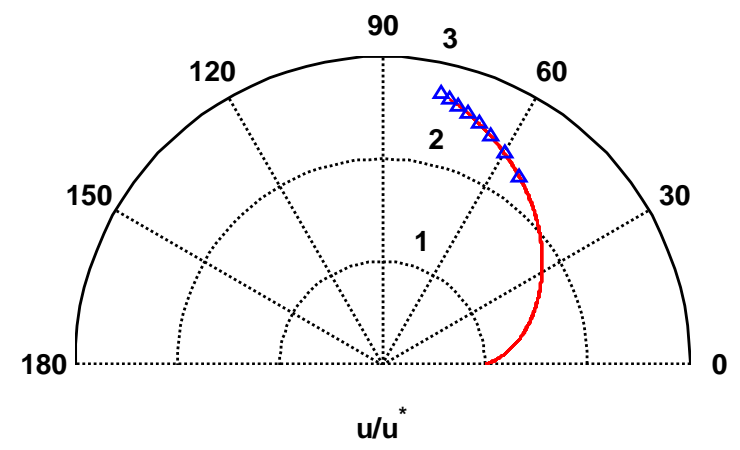

(a)

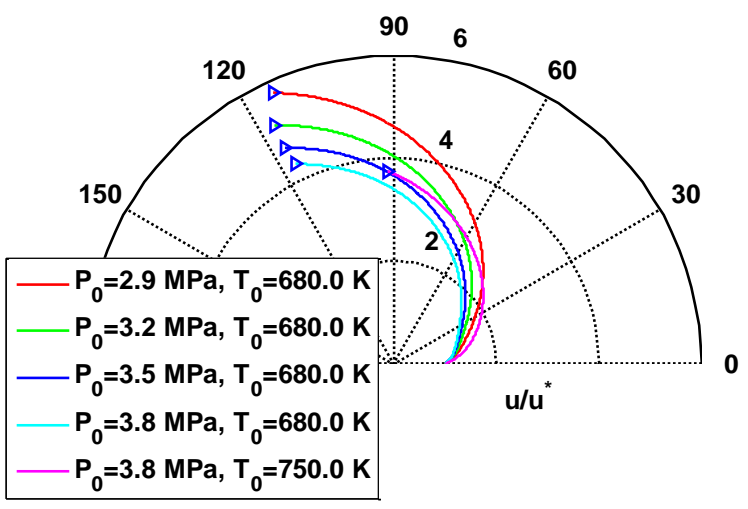

(b)

Figure 8 (a) Prandtl-Meyer angle - normalized Mach number polar diagram of $\gamma=1.03$ perfect gas. (b) diagram of dodecane's jet. 


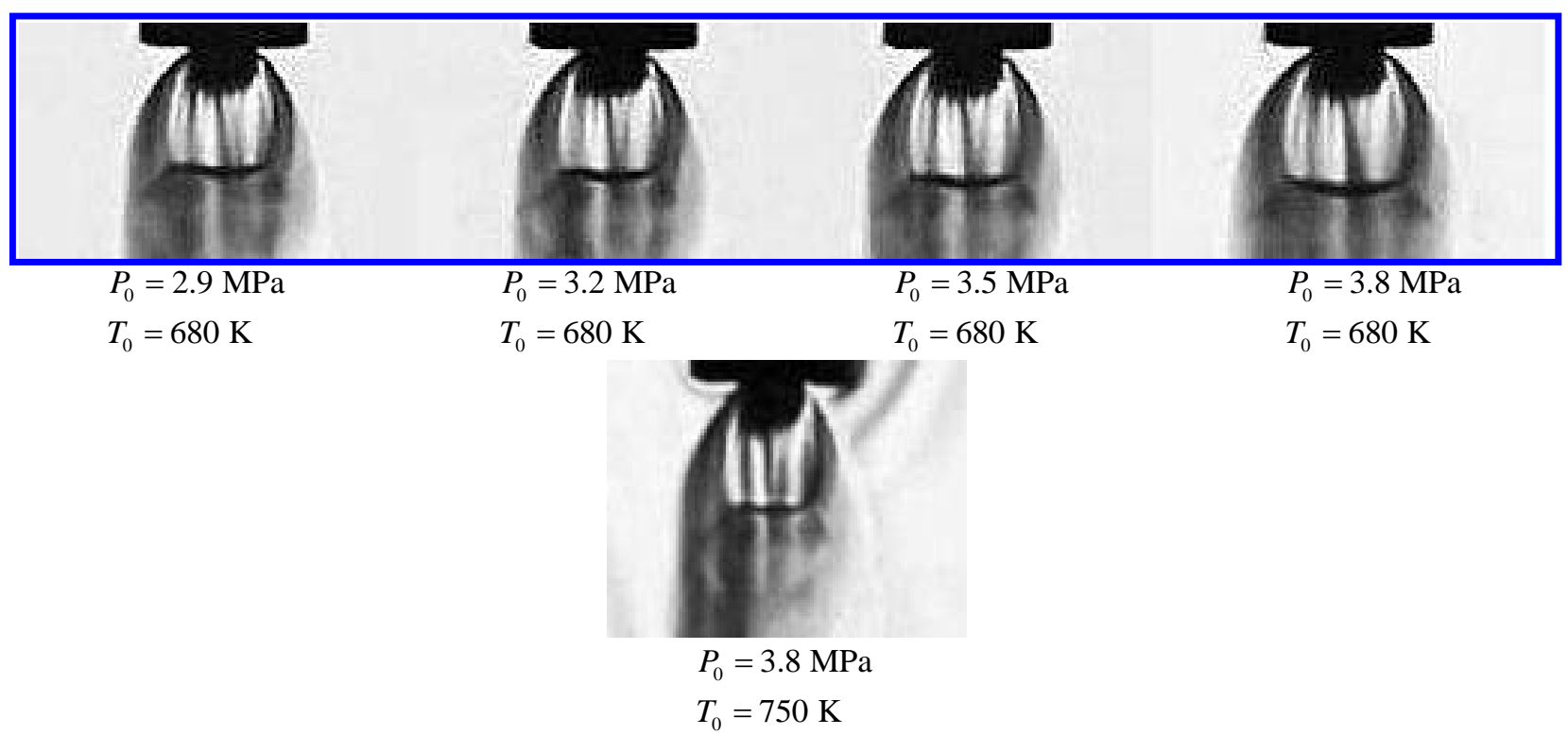

Figure 9. Supercritical kerosene sonic jet into quiescent atmosphere at different pressures and temperatures. The diameter of orifice is $2.671 \mathrm{~mm}$, outer diameter is $8.8 \mathrm{~mm}$. temperature dependence is observed.

However, due to the limitation of resolution of the photograph, measurement of jet angle at the vicinity of the orifice edge is impossible, but the trend is obvious. Larger shock cell comes with higher total pressure, and the shock cell size decreased with increasing temperature. The results showed in Figure 9 can be interpreted by Figure 8(b) qualitatively. Using ideal gas theory, the contraction of underexpanded jet shock cell at higher temperature with same pressure ratio cannot be explained, as shown in Figure 8.

\section{Conclusion}

The differential equations of steady quasi-1D flow with friction and heat addition were formulated for arbitrary equation of state to study dense gas behavior of hydrocarbon fuels. The equations are fully simplified using Bridgman's table. By introducing two new non-dimensional numbers $g$ and $X$, a complete set of non-dimensional numbers to characterize the dense gas dynamics are identified, classified and discussed. A new numerical method based on conservation laws is proposed to study isentropic flow and shock wave of dense gas and applied to flows of $\mathrm{n}$-dodecane. And several conclusions can be drawn from the analysis:

1. If $g=\rho \mu_{j} C_{p} \rightarrow 0$, and defining $\hat{R}=C_{p}-C_{v}$, the first order derivative related equations for ideal gas dynamics is quantitatively valid for dense gas formally.

2. If $\Gamma>1$ and $g>-1$, the differential relations for ideal gas dynamics is qualitatively valid for dense gas in isentropic flow. To n-dodecane, if the stagnation point resides sufficiently far from the critical point (e.g. above $2.5 p_{c}$ and $1.2 T_{c}$ ), ideal gas relations is qualitatively valid except the sound speed relationship eq.(18) If $\Gamma<1$ or $g<-1$, some prediction of ideal gas dynamics might be qualitatively incorrect for dense gas.

3. For a quasi-1D flow without friction and heat addition, the maximum momentum flux is reached at Mach number of $\sqrt{2}$ for arbitrary fluid. This result might be useful to design the optimal fuel injector.

4. To arbitrary fluid, stagnation pressure always decreases after shock wave, while the stagnation temperature variation depends upon the sign of Joule-Thomson coefficient.

5. Temperature dependence of supercritical kerosene jet structure can be partially interpreted by Prandtl-Meyer expansion of dense gas. And it is clarified that no condensation occurs in isentropic acceleration of supercritical kerosene jet, because kerosene is dry fluid. 


\section{Appendix: Detailed deduction of differential formulae of quasi-1D flow}

Diagram of the deduction is as follows.

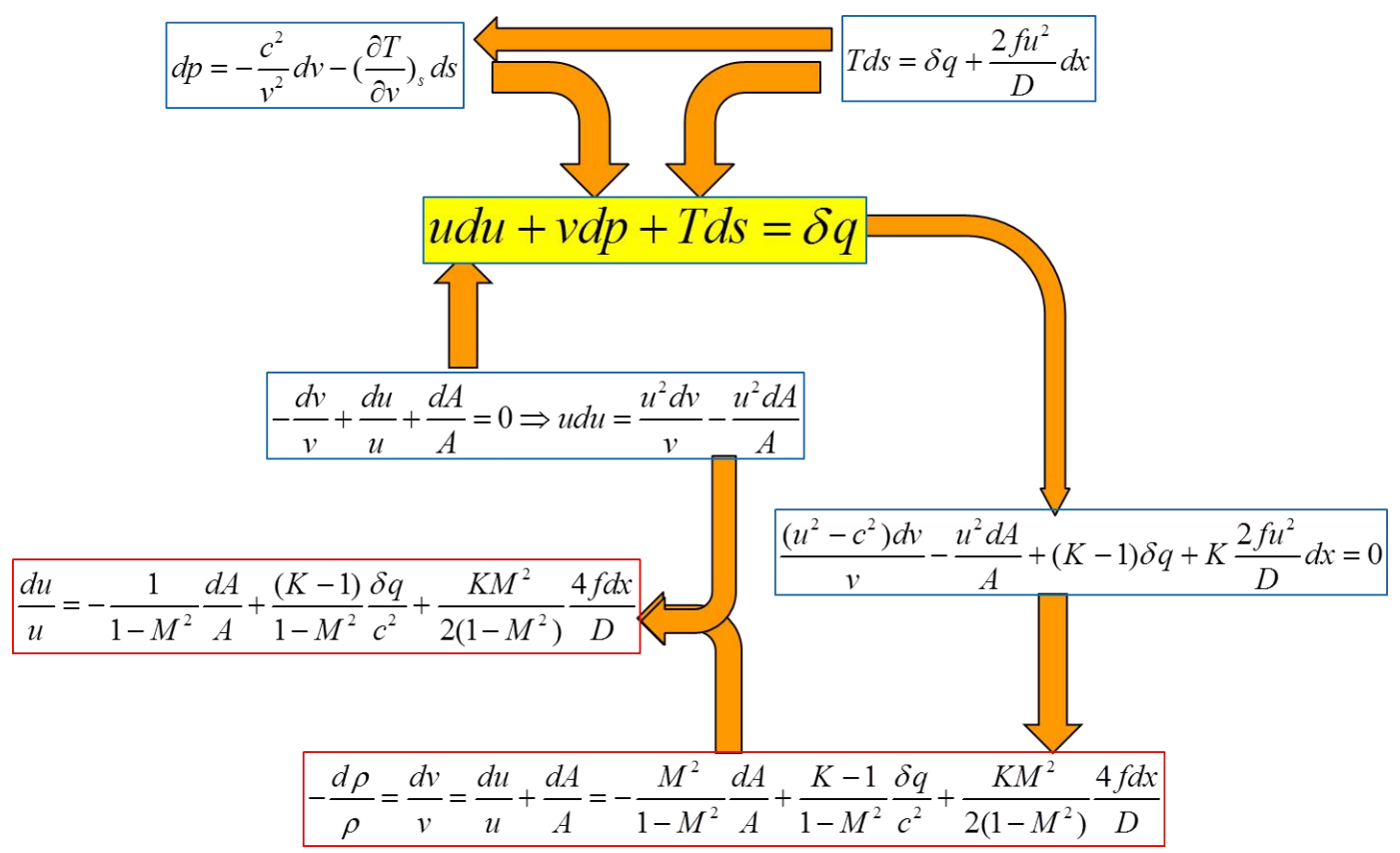

Figure 10 Deduction of first order derivative related differential relations. Conclusion is in red box, predefined formula is in blue box.

Then, with $d s, d v$ expressions, we can derive any thermodynamic property's formula via:

$$
d X=\left(\frac{\partial X}{\partial v}\right)_{s} d v+\left(\frac{\partial X}{\partial s}\right)_{v} d s
$$

That is how $d T, d c$ expressions are obtained. A collection of the formulae is in Table 3.

Table 3 table of influence coefficients of quasi-1D dense gas dynamics

\begin{tabular}{|c|c|c|c|}
\hline & $\frac{d A}{A}$ & $\frac{\delta q}{c^{2}}$ & $\frac{4 f d x}{D}$ \\
\hline$\frac{d \rho}{\rho}=-\frac{d v}{v}$ & $\frac{M^{2}}{1-M^{2}}$ & $-\frac{K-1}{1-M^{2}}$ & $-\frac{K M^{2}}{2\left(1-M^{2}\right)}$ \\
\hline$\frac{d u}{u}$ & $-\frac{1}{1-M^{2}}$ & $\frac{(K-1)}{1-M^{2}}$ & $\frac{K M^{2}}{2\left(1-M^{2}\right)}$ \\
\hline$\frac{d p}{p}$ & $k_{s} \cdot \frac{M^{2}}{1-M^{2}}$ & $-k_{s} \cdot \frac{(K-1) M^{2}}{1-M^{2}}$ & $-k_{s} \cdot \frac{M^{2}}{2} \cdot \frac{(K-1) M^{2}+1}{1-M^{2}}$ \\
\hline$\frac{d T}{T}$ & $\frac{(K-1) M^{2}}{1-M^{2}}$ & $\frac{(K-1)^{2}}{\gamma-\gamma} \frac{1-\gamma M^{2}}{1-M^{2}}$ & $\frac{M^{2}}{2} \frac{(K-1)\left(K-\gamma-\gamma(K-1) M^{2}\right)}{(\gamma-1)\left(1-M^{2}\right)}$ \\
\hline$\frac{d c}{c}$ & $\frac{M^{2}(\Gamma-1)}{1-M^{2}}$ & $\frac{(K-1)(1-\Gamma)}{1-M^{2}}+X$ & $\frac{M^{2}}{2}\left(\frac{K(1-\Gamma)}{\left(1-M^{2}\right)}+X\right)$ \\
\hline$\frac{d M}{M}$ & $-\frac{1+M^{2}(\Gamma-1)}{1-M^{2}}$ & $\frac{(K-1) \Gamma}{1-M^{2}}-X$ & $\frac{M^{2}}{2}\left(\frac{K \Gamma}{\left(1-M^{2}\right)}-X\right)$ \\
\hline
\end{tabular}

All the coefficients are expressed in terms of non-dimensional numbers listed in Table 1. Some important relationship in the deduction is listed here: 
- If we choose $C_{p}, \beta, \kappa$ as primary $1^{\text {st }}$ order derivatives, as Bejan's book used: ${ }^{29}$

$$
\begin{gathered}
\mu_{j}=\frac{v}{C_{p}}(\beta T-1) \\
C_{v}=C_{p}-\frac{\beta^{2}}{\kappa} v T \\
c^{2}=-\frac{v C_{p}}{\beta^{2} v T-\kappa C_{p}} \\
g=\beta T-1 \\
K=\frac{\beta v}{\kappa C_{p}-\beta^{2} v T}+1
\end{gathered}
$$

- If we choose $\mu_{j}, C_{p}, C_{v}$ as primary $1^{\text {st }}$ order derivatives:

$$
\begin{gathered}
g=\rho C_{p} \mu_{j} \\
\beta=\frac{g+1}{T} \\
\kappa=\frac{v}{\left(C_{p}-C_{v}\right) T}(g+1)^{2} \\
c^{2}=\frac{\gamma\left(C_{p}-C_{v}\right) T}{(g+1)^{2}} \\
K=\frac{\gamma-1}{g+1}+1
\end{gathered}
$$

- If we choose $c^{2}, C_{p}, C_{v}$ as primary $1^{\text {st }}$ order derivatives:

$$
g=\frac{ \pm \sqrt{\gamma\left(C_{p}-C_{v}\right) T}}{c}-1
$$

In supercritical and gaseous region, the sign is always positive.

$$
\begin{gathered}
\beta=\frac{\sqrt{\gamma\left(C_{p}-C_{v}\right) T}}{c T} \\
\kappa=\frac{\gamma}{\rho c^{2}} \\
K=\frac{\gamma-1}{g+1}+1
\end{gathered}
$$

- If we choose $c^{2}, C_{p}, \mu_{j}$ as primary $1^{\text {st }}$ order derivatives, as in Table 3:

$$
\begin{gathered}
C_{v}=\frac{C_{p}^{2}}{C_{p}+c^{2}(g+1)^{2}} \\
\gamma=1+\frac{c^{2}(g+1)^{2}}{C_{p}} \\
\beta=\frac{g+1}{T} \\
\kappa=\frac{\gamma}{\rho c^{2}} \\
K=\frac{\gamma-1}{g+1}+1
\end{gathered}
$$




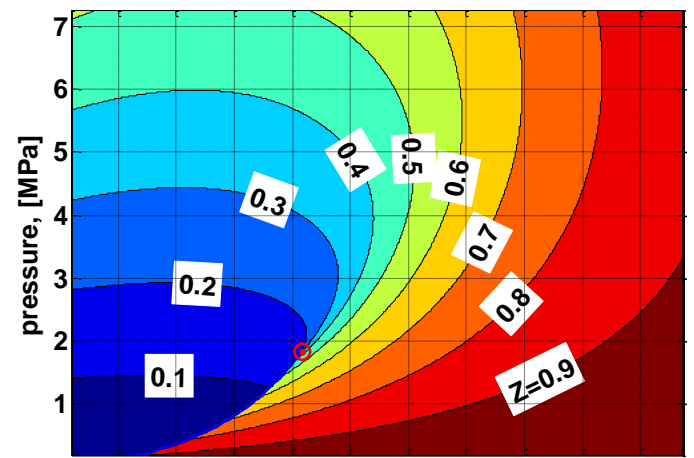

500550600650700750800850900950 temperature, [K]

(a) Compressibility Factor

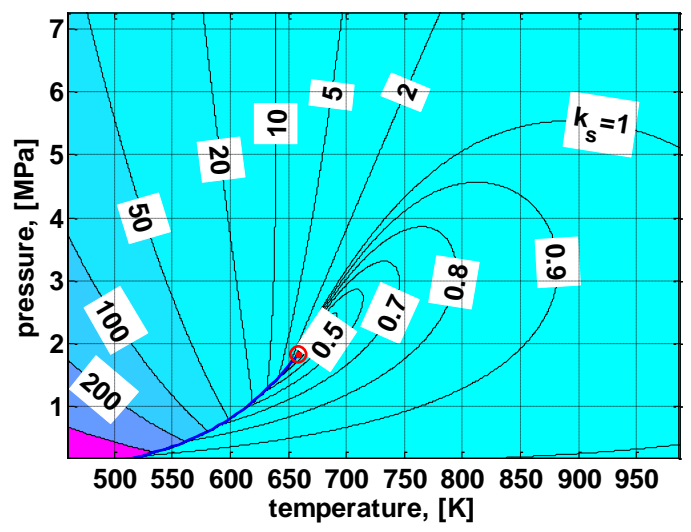

(c) General isentropic exponent

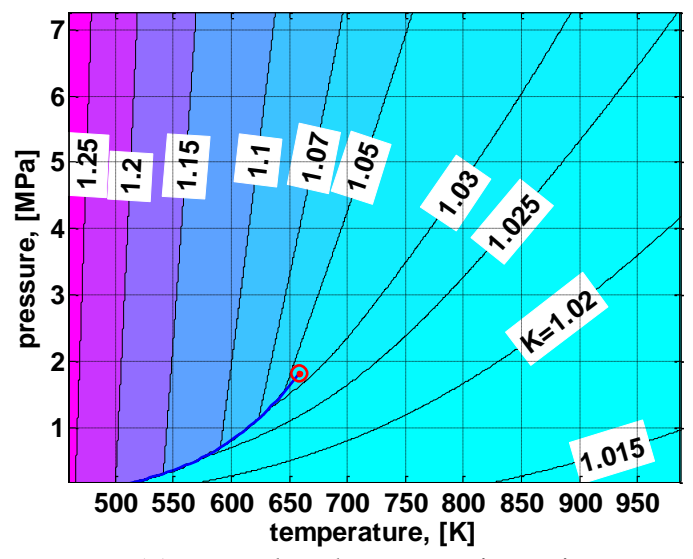

(e) Equvalent heat capacity ratio

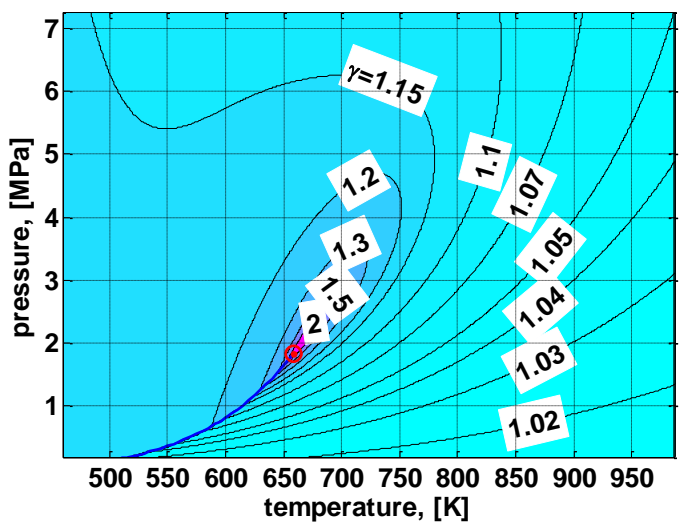

(b) Heat capacity ratio

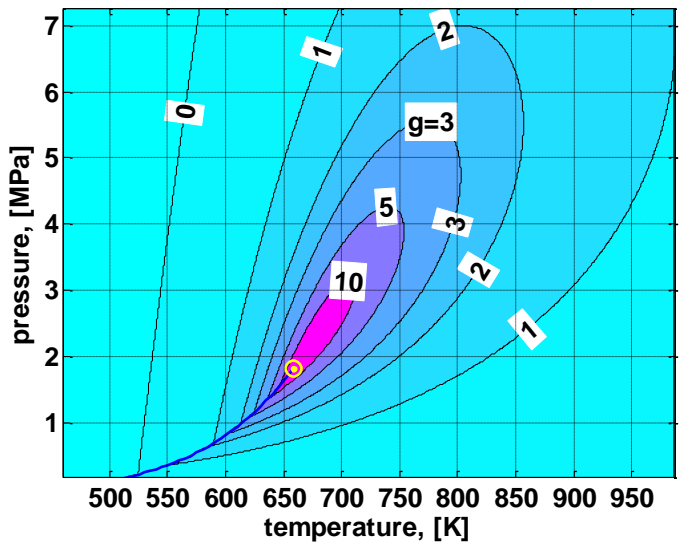

(d) Non-dimensional Joule-Thompson Coeffcient

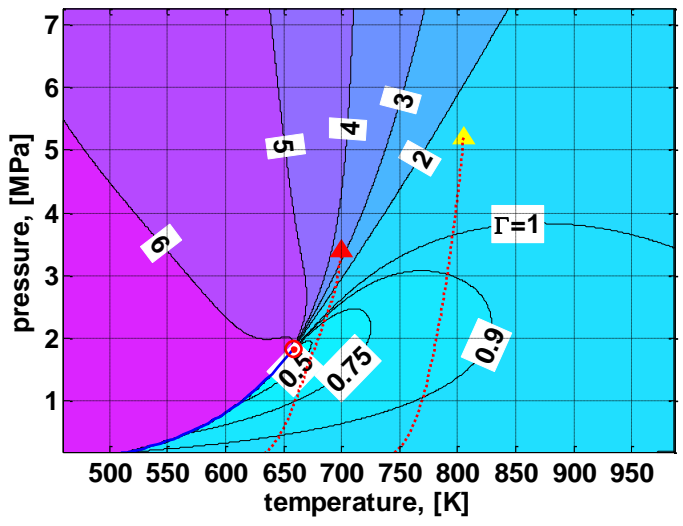

(f) Fundamental derivative of gas dynamics 


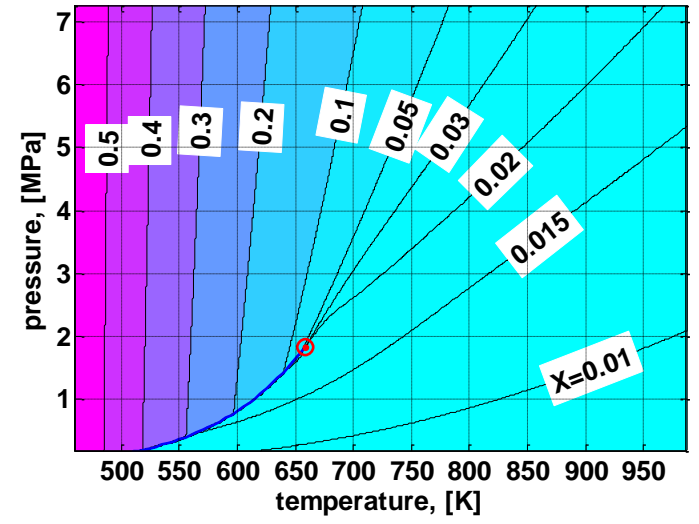

(g) Newly introduced non-dimentional number $X$

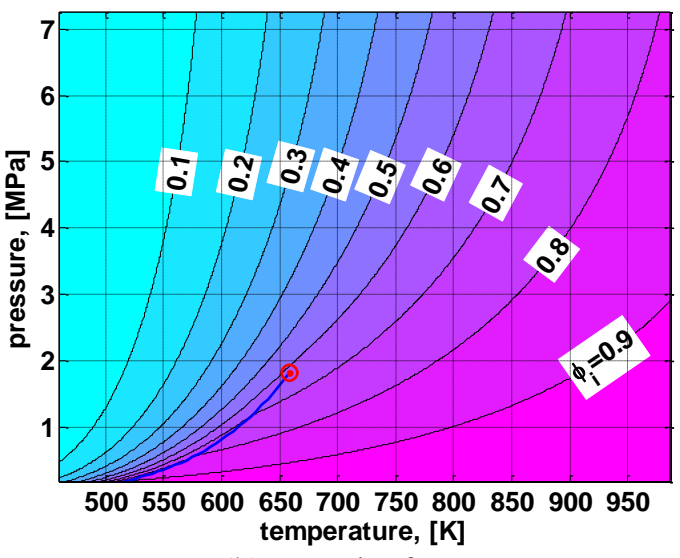

(h) Fugacity factor

Figure 11. Contour of non-dimensional numbers mentioned in this paper. The fluid is dodecane.

\section{Acknowledgments}

Current research program of the Chinese Academy of Science was supported by the National Natural Science Foundation of China under contractor No. 91016005 and No. 10621202. The authors would like to acknowledge Prof. Gong Yu, for discussions on ideal gas dynamics.

\section{References}

${ }^{1}$ Fan, X. J., Yu, G., Li, J. G., Zhang, X. Y. and Sung, C. J., "Investigation of Vaporized Kerosene Injection and Combustion in a Supersonic Model Combustor," Journal of Propulsion and Power, Vol. 22, No. 1, 2006, pp. 103-110.

${ }^{2}$ Li, X., Zhong, F., Fan, X., Huai, X. and Cai, J., "Study of Turbulent Heat Transfer of Aviation Kerosene Flows in a Curved Pipe at Supercritical Pressure," Applied Thermal Engineering, Vol. 30, No. 13, 2010, pp. 1845-1851.

${ }^{3}$ Harinck, J., Turunen-Saaresti, T., Colonna, P., Rebay, S. and Van Buijtenen, J., "Computational Study of a High-Expansion Ratio Radial Organic Rankine Cycle Turbine Stator," Journal of Engineering for Gas Turbines and Power, Vol. 132, No. 5, 2010, pp. 054501-054506.

${ }^{4}$ Chen, H., Goswami, D. Y. and Stefanakos, E. K., "A Review of Thermodynamic Cycles and Working Fluids for the

Conversion of Low-Grade Heat," Renewable and Sustainable Energy Reviews, Vol. 14, No. 9, 2010, pp. 3059-3067.

${ }^{5}$ Thompson, P., "A Fundamental Derivative in Gasdynamics," Phys. Fluids, Vol. 14, No. 9, 1971, pp. 1843.

${ }^{6}$ Kouremenos, D. A., "The Normal Shock-Waves of Real Gases and the Generalized Isentropic Exponents," Forschung Im Ingenieurwesen-Engineering Research, Vol. 52, No. 1, 1986, pp. 23-31.

${ }^{7}$ Cramer, M., "Steady, Isentropic Flows of Dense Gases," Phys. Fluids A, Vol. 3, No. 1, 1991, pp. 219.

${ }^{8}$ Schnerr, G. H. and Leidner, P., "Diabatic Supersonic Flows of Dense Gases," Physics of Fluids a-Fluid Dynamics, Vol. 3 , No. 10, 1991, pp. 2445-2458.

${ }^{9}$ Schnerr, G. H. and Molokov, S., "Exact Solutions for Transonic Flows of Dense Gases in Two-Dimensional and

Axisymmetric Nozzles," Physics of Fluids, Vol. 6, No. 10, 1994, pp. 3465-3472.

${ }^{10}$ Stojkovic, D., Djordjevic, V. D. and Cvijanovic, P. S., "On the Effect of Friction in Steady Flow of Dense Gases in Pipes,"

International Journal of Heat and Fluid Flow, Vol. 22, No. 4, 2001, pp. 480-485.

${ }^{11}$ Thompson, P. A., Compressible-Fluid Dynamics, Advanced Engineering Series, Rensselaer Polytechnic Institute Press, 1988.

${ }^{12}$ Congedo, P. M., Corre, C. and Cirmella, P., "Airfoil Shape Optimization for Transonic Flows of Bethe-Zel'dovich- Thompson Fluids," Aiaa Journal, Vol. 45, No. 6, 2007, pp. 1303-1316.

${ }^{13}$ Cinnella, P. and Congedo, P. M., "Aerodynamic Performance of Transonic Bethe-Zel'dovich-Thompson Flows Past an Airfoil," Aiaa Journal, Vol. 43, No. 2, 2005, pp. 370-378.

${ }^{14}$ Colonna, P. and Rebay, S., "Numerical Simulation of Dense Gas Flows on Unstructured Grids with an Implicit High

Resolution Upwind Euler Solver," International Journal for Numerical Methods in Fluids, Vol. 46, No. 7, 2004, pp. 735-765.

${ }^{15}$ Nannan, N. R., Zamfirescu, C. and Colonna, P. M., "Detailed Design of the Flexible Asymmetric Shock Tube (Fast) Facility at the P\&E Department of the Delft University of Technology", ET-2263, 2007.

${ }^{16}$ Colonna, P., Guardone, A. and Nannan, N. R., "Siloxanes: A New Class of Candidate Bethe-Zel'dovich-Thompson Fluids," Physics of Fluids, Vol. 19, No. 8, 2007

${ }^{17}$ Bridgman, P. W., "A Complete Collection of Thermodynamic Formulas.," Physical Review, Vol. 3, No. 4, 1914, pp. $273-281$.

${ }^{18}$ Ely, J. F. and Huber, M. L., "Nist Thermophysical Properties of Hydrocarbon Mixtures Database (Supertrapp). Nist Standard Reference Database 4.", 1990.

${ }^{19}$ Shapiro, A. H., Dynamics and Thermodynamics of Compressible Fluid Flow, Reprint, R.E. Krieger, 1983 
${ }^{20}$ Portz, R. and Segal, C., "Penetration of Gaseous Jets in Supersonic Flows," Aiaa Journal, Vol. 44, No. 10, 2006, pp. 24262429.

${ }^{21}$ Gruber, M. R., Nejad, A. S. and Dutton, J. C., "An Experimental Investigation of Transverse Injection from Circular and Elliptical Nozzles into a Supersonic Crossflow", 1996.

${ }^{22}$ Billig, F. S. and Schetz, J. A., "Penetration of Gaseous Jets Injected into a Supersonic Stream (Penetration of Gaseous Jets Injected into Supersonic Stream Treated by Solid Body Drag Model, Noting Application to Hypersonic Ramjet Combustion Chamber)," JOURNAL OF SPACECRAFT AND ROCKETS, Vol. 3, No. 1966, pp. 1658-1665.

${ }^{23}$ Hendricks, R. C., Peller, I. C. and Baron, A. K., Joule-Thomson Inversion Curves and Related Coefficients for Several Simple Fluids, National Aeronautics and Space Administration, 1972.

${ }^{24}$ Anderson, J. D., Hypersonic and High-Temperature Gas Dynamics, $2^{\text {nd }}$ ed., American Institute of Aeronautics and Astronautics, 2006

${ }^{25}$ Mcbride, B. and Gordon, S., "Computer Program for Calculation of Complex Chemical Equilibrium Compositions and Applications", N96-32038 (AH), 1996.

${ }^{26}$ Yang, M., "High Speed Pulsed Schlieren Technology and Its Application to Flow Visualization in Supersonic Combustor (in Chinese)," Master's Dissertation, State Key Laboratory of High temperature gas dynamics, Institute of Mechanics, Beijing, 2012.

${ }^{27}$ Zhong, F. Q., Fan, X. J., Wang, J., Yu, G. and Li, J. G., "Characteristics of Compressible Flow of Supercritical Kerosene," Acta Mechanica Sinica, Vol. 28, No. 1, 2012, pp. 8-13.

${ }^{28}$ Deng, H. W., Zhang, C. B., Xu, G. Q., Tao, Z., Zhang, B. and Liu, G. Z., "Density Measurements of Endothermic Hydrocarbon Fuel at Sub- and Supercritical Conditions," Journal of Chemical and Engineering Data, Vol. 56, No. 6, 2011, pp. 2980-2986.

${ }^{29}$ Bejan, A., Advanced Engineering Thermodynamics, 3rd, John Wiley \& Sons, 2006. 